EUROPEAN ORGANIZATION FOR NUCLEAR RESEARCH

European Laboratory for Particle Physics

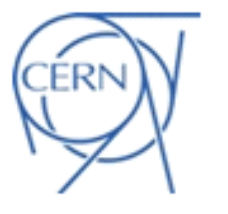

sLHC Projects

sLHC Project Report 48

\title{
A MEASUREMENT SYSTEM FOR FAST-PULSED MAGNETS: A CASE STUDY ON LINAC4 AT CERN
}

\author{
P. Arpaia ${ }^{1,2}$, M. Buzio 2 , O. Dunkel², D. Giloteaux², G. Gollucio ${ }^{1,2}$, \\ 1 Department of Engineering, University of Sannio, Benevento, Italy \\ 2 CERN, Geneva, Switzerland
}

\begin{abstract}
A new magnetic measurement system for validating and characterizing permanent and fast-pulsed iron-dominated magnets is presented. This system is going to be used on the magnet series tests of the new linear particle accelerator Linac 4 of the Large Handron Collider at the European Organization for Nuclear Research (CERN) to perform a complete field analysis of the entire acceleration line. Measurement results will be useful to tune better the beam brightness during the first acceleration step. In this paper, the requirements, the architecture, and the most critical parts of the design are highlighted. Then, the preliminary experimental results obtained over the first Linac 4 magnet prototypes are discussed.
\end{abstract}

Linac4 


\title{
A Measurement System for Fast-Pulsed Magnets: a case study on Linac4 at CERN
}

\author{
Pasquale Arpaia ${ }^{1,2}$, Marco Buzio², Olaf Dunkel ${ }^{2}$, \\ David Giloteaux ${ }^{2}$, Giancarlo Golluccio ${ }^{1,2}$ \\ ${ }^{1)}$ Department of Engineering, University of Sannio, \\ Corso Garibaldi 107, 82100 Benevento, Italy. $\mathrm{Ph}:+39$ \\ 0824305804-17, Fax: +390824305840, \\ E-mail: arpaia@unisannio.it \\ ${ }^{2)}$ CERN, European Organization for Nuclear Research,1211 \\ Geneva 23, Switzerland. Ph: +41 227676111 , \\ E-mail: giancarlo.golluccio@cern.ch;
}

\begin{abstract}
A new magnetic measurement system for validating and characterizing permanent and fast-pulsed iron-dominated magnets is presented. This system is going to be used on the magnet series tests of the new linear particle accelerator Linac 4 of the Large Handron Collider at the European Organization for Nuclear Research (CERN) to perform a complete field analysis of the entire acceleration line. Measurement results will be useful to tune better the beam brightness during the first acceleration step. In this paper, the requirements, the architecture, and the most critical parts of the design are highlighted. Then, the preliminary experimental results obtained over the first Linac 4 magnet prototypes are discussed.
\end{abstract}

Keywords- Accelerator magnets, Permanent magnets, Magnetic field measurement.

\section{INTRODUCTION}

The preparation of the CERN injectors for the LHC allowed the prefixed goals to be reached, but at the same time showed clearly the achievement of their limits, in terms of both brightness and intensity. A new linear particle accelerator, Linac 4, is currently being built at CERN for replacing the existing Linac 2 to provide a high-intensity, high-quality beam source for LHC [1]. This machine includes a large number of narrow-aperture quadrupole electromagnets (EMQ) in order to keep the beam focused along the acceleration path. These magnets must provide a high gradient (of the order of $20 \mathrm{~T} / \mathrm{m}$ ) in a very limited space, and are therefore powered with relatively high currents up to $200 \mathrm{~A}$. The transversal focusing of the beam is achieved in the Drift Tube Linac by including several Permanent Magnet Quadrupoles (PMQ) allowing gradients and RF efficiency higher than the electromagnetic quadrupole ones, owing to their compactness. The specification compliance of the magnets is provided by suitable magnetic acceptance tests. They are aimed at (i) validating magnet design calculations and manufacturing procedures; (ii) ensuring the manufactured units to remain within the specified tolerances, thus allowing CERN to accept or reject the quadrupoles; (iii) running summaries and statistics to identify production trends; (iv) characterizing in detail the magnets in view of their use in the machine, translating beam optics requirements in term of practical measurements; and (v) ensuring long-term exploitability of results in view of future changes and upgrades.

For these motivations, a new measurement facility to test a series of PMQ and EMQ magnets is under development at CERN in collaboration with the University of Sannio. The system has to perform (i) the classical rotating coil measurement for permanent magnets, and (ii) a stepped fixed coil measurement (integrated flux measured sequentially at $\mathrm{N}$ equally spaced azimuthal coil positions) for very-fast ramped fields. The stepped fixed coil method is already well-known [2], but the encoder hardware and the coil design limited the attainable accuracy. Another solution to measure the harmonic content of fast changing fields in multipoles is a stationary coil array [3]-[4]. The array is made of several printed circuit coils mounted on a cylindrical structure, e.g. 32 coils to measure up the $16^{\text {th }}$ harmonic of the field. This technique requires highprecision angular positioning on the support and all coils to be identical, thus it turns out to be difficult and expensive for narrow aperture magnets.

In this paper, by exploiting the stepped coil approach, a new magnetic measurement system for validating and characterizing permanent and fast-pulsed iron-dominated magnets is proposed. In Sections 2 and 3, the requirements and the architecture of the proposed system are respectively described. In Section 4, the design of the most critical parts of the system, namely the coil and the encoder, is illustrated. In Section 5, the preliminary experimental results obtained by measuring the spare PMQ quadrupole are discussed.

\section{SYSTEM REQUIREMENTS}

The main design objectives of the new magnetic measurement system are (i) to create a system to measure small magnets with a bore diameter in the range $(20,40) \mathrm{mm}$ which, respect to the existing system developed in the 1980s for Linac2, guarantees long-term reliability and maintainability and (ii) guarantee the long-term possibility to measure also the spare magnets and replacements during the life time of the LHC [5].

With this aim, the following requirements, arising from beam optics, have to be fulfilled: 
- determine the main magnetic field polarity;

- measure the integral field strength and direction (vs. current excitation where applicable);

- measure the field quality in terms of both integral harmonics (up to 10) and position of the magnetic axis;

- carry out a complete harmonic analysis for narrow-aperture permanent magnets.

Specifically, for pulsed measurements it is necessary to:

- specify the field repeatability (history dependence and power supply stability);

- measure the eddy current effects;

- analyze quadrupoles with a high angular accuracy.

Furthermore, the entire system should have a polyvalent harmonic coil concept to measure permanent and pulsed quadrupoles in continuous or stepwise rotation mode, interchangeable magnet supports, and optimized dipole and quadrupole-compensated harmonic coils able to measure the field quality up to the requested order. For the pulsed mode, a high resolution positioning system is required in order to reach the same harmonic resolution within a few $\mu \mathrm{rad}$. The system has to be able to achieve an accurate azimuthal positioning.

\section{ARCHITECTURE}

The classical flux measurement based on rotating coils [6] is realized by using a stepping motor in continuous rotation and by acquiring compensated and absolute signals through a Fast Digital Integrator (FDI) [7], guaranteeing a bandwidth of $250 \mathrm{kHz}$ and typical signal-to-noise ratio of $105 \mathrm{~dB}$. A CERN proprietary angular encoder card (PXI-based trigger card) [8] provides the trigger signal to the acquisition cards up to 131,072 points per revolution.

The same hardware configuration can be used to carry out measurements on a fast pulsed magnet. In Figure 1, the architecture of the proposed system is shown. The acquisition must be synchronized with a very-short current pulse of about $1 \mathrm{~ms}$, generated by the current supply. For each angular position, the voltage generated by the magnetic flux linked to the coil is integrated by the FDIs between two trigger events. The first trigger is synchronized with the start of the current's ramp; the second trigger for the stop is provided by a timing card, with a programmable constant delay (usually synchronized with the end of the current flat top).

The system is controlled by a software program developed by means of the new Flexible Framework for Magnetic Measurements (FFMM [9]), developed at CERN in cooperation with the University of Sannio, providing an easy way to change both the measurement procedure and configuration.

At each step, proper processing and normalization for the gains of the amplifier's chain (implemented in the FDI firmware directly) are carried out.

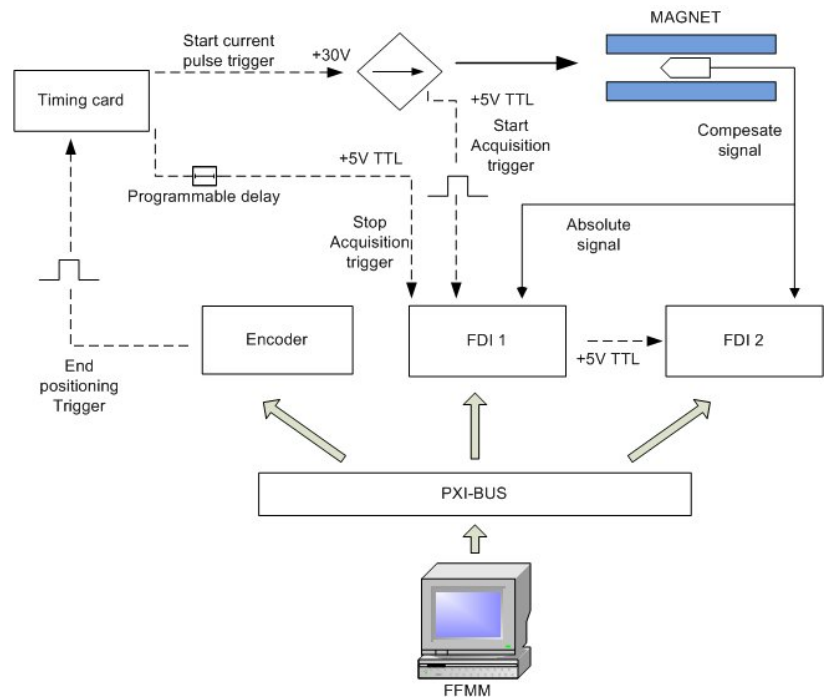

Figure 1: architecture of the proposed measurement system.

The classical harmonic analysis is applied to the acquired data [10], the value of the magnetic flux $\Phi\left(\theta_{k}\right)$, as a function of the angle $\theta_{k}$, at a discrete series of angular positions $\mathrm{k}$ is delivered for a total of $\mathrm{N}$ points. The sampling points are equally spaced over the interval $[0, \ldots, 2 \pi]$. The field harmonic content is proportional to the DFT of the flux samples:

$$
\Psi_{n}=\sum_{k=1}^{N} \Phi_{k} e^{-2 \pi i(n-1) \frac{(k-1)}{n}}
$$

From these complex values of the DFT, the harmonic coefficients can be derived [10]:

$$
C_{n} \approx \frac{2}{N} \frac{1}{L} \frac{n R_{r e f}^{n-1}}{\chi_{n}} \Psi_{n+1}
$$

where $L$ is the length of the coil, $n$ the harmonic order, $R_{r e f}$ the reference radius (a constant value $0.0075 \mathrm{~m}$ ), and $\chi_{n}$ are complex geometric correction factors related to the coil sensitivity.

\section{MAIN COMPONENT DESIGN}

In the following, the design of the most critical parts of the proposed measurement system, (i) the angular encoder, and (ii) the flux coil, is illustrated.

\section{A. Angular Encoder}

One of the main design challenges was to build up a unique structure for carrying out both rotating and fixed coil measurements [7] with an angular accuracy of $\pm 100 \mu \mathrm{rad}$. The PXI-based trigger card provides a direct interface with the stepping motor, encoder and tilt sensor servo-control. The card also manages the encoder pulses to trigger in rotating coil mode the FDI acquisition. The encoder used is a ZH-65 
encoder from $\mathrm{T}+\mathrm{R}$ electronics ${ }^{\circledR}$, with both incremental and absolute position output. The incremental output has two quadrature channels (90 degrees out of phase), provides an angular reference with a resolution of $25 \mu \mathrm{rad}$ and is the trigger reference for the acquisition of the continuously rotating coil. The absolute output is used in stepwise mode and has 17 bit of resolution (corresponding to $48 \mu \mathrm{rad}$ ) with a standard Synchronous Serial Interface (SSI). All angular measurements can be referred to gravity through an electrolytic inclinometer positioned on the coil shaft, sampled by an ADC integrated in the encoder board. The systematic error due to misalignment between the encoder zero and the gravity reference is removed by repeating magnetic measurements after having flipped the magnet around a vertical axis [11]. The mechanics was conceived to easily disconnect the motor head from the coil, in order to flip or replace the magnet, by maintaining the angular reference (the encoder) simultaneously. The system is also conceived to cancel out the systematic offsets affecting the magnet axis measurement by flipping the magnet around a horizontal axis.

\section{B. Harmonic Coil}

The design of the harmonic coil is based [12]. Figure 2 shows the harmonic coil configuration. It presents two nested radial windings on the same plane with the same effective surface linked to the flux and the same central radius. The external one (ABS coil) generates the absolute signal relative to the main field of the quadrupole. The nested one (CMP coil) is used in opposition series to the external coil to generate a signal more sensitive to the high order harmonics. In this configuration, the two coils must have the same equivalent surface, thus the internal winding, since it has half of the surface has twice the number of turns.

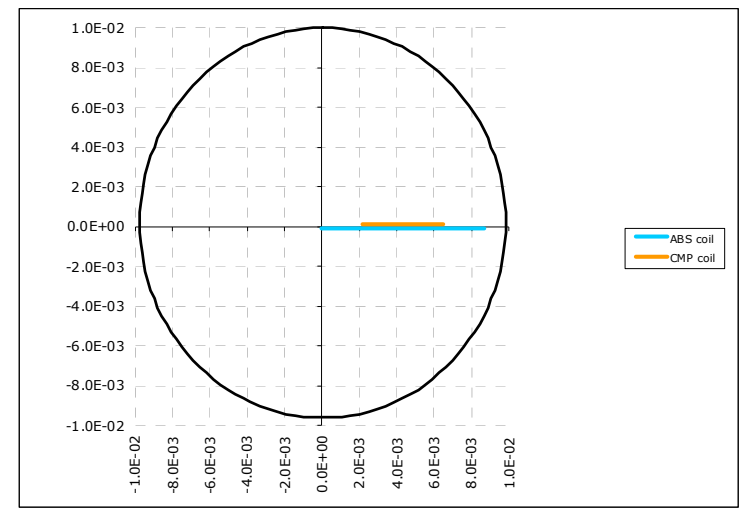

Figure 2: design of the coil section.

In Figure 3, details about the coil prototyping are reported.

In Table 1, the main parameters of the shaft after the calibration are reported [13].
Table 1: coils parameters

\begin{tabular}{|c|c|c|}
\hline & $\begin{array}{c}\text { External } \\
\text { coil A }\end{array}$ & $\begin{array}{c}\text { Internal } \\
\text { Coil B }\end{array}$ \\
\hline Length [m] & 0.225485 & 0.199970 \\
\hline N turns & 32 & 64 \\
\hline Center radius [m] & 0.003621 & 0.003563 \\
\hline Width [m] & 0.008769 & 0.004672 \\
\hline Total Area [m2] & 0.063273 & 0.059798 \\
\hline R max [m] & 0.008005 & 0.005899 \\
\hline Rmin[m] & -0.000764 & 0.001227 \\
\hline & & \\
\hline & &
\end{tabular}

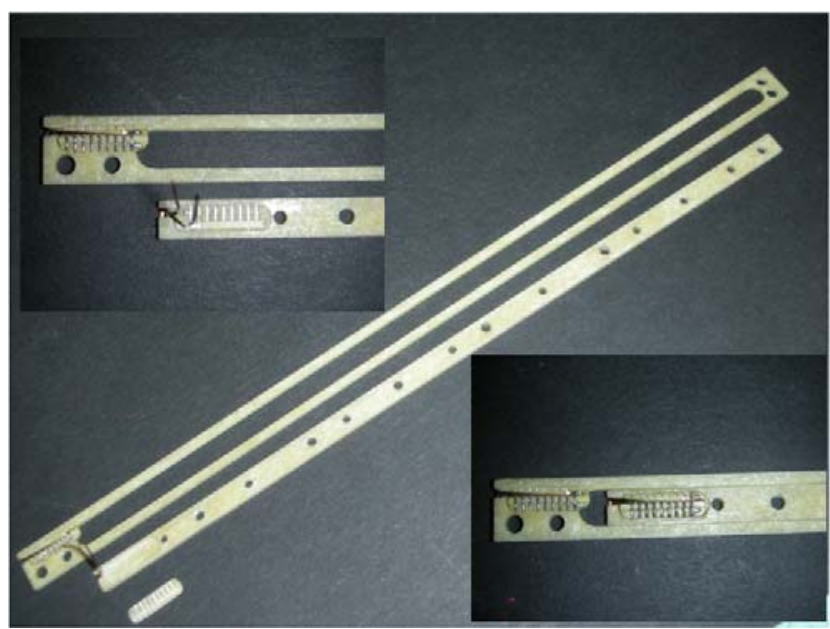

Figure 3: coil prototyping.

\section{EXPERIMENTAL RESULTS}

In the following, the experimental results of (i) the encoder tests, and (ii) the tests on prototype a permanent magnet of the drift tube, are illustrated.

\section{A. Encoder Tests}

Specific tests were carried out to check the performance of the positioning system. The system needs less than 1 minute to align the shaft to the gravity. Once the control system find the zero of the inclinometer, (with an accuracy of $\pm 25 \mu \mathrm{rad}$, corresponding to half of the ADC's LSB) the absolute encoder is set to the origin, a complete turn was divided in 16384 steps, and the position read by the encoder is compared to the ideal value (with $48 \mu \mathrm{rad}$ or $0.0027^{\circ}$ step resolution). The maximum positioning error is less than $0.002^{\circ}$. However, an error of a single encoder step occurs in some positions due probably to the stop of the motor on the encoder step or to the table support being not perfectly aligned. Nevertheless, the uncertainty is less than the requirements $\left( \pm 0.0057^{\circ}\right)$. The time needed for the system to complete the whole turn in 64 points is less than 10 minutes.

\section{B. Preliminary Tests on Drift Tube Linac Prototype Permanent Magnet}

The system was tested on a prototype of permanent magnet $45 \mathrm{~mm}$ long, with $\varnothing 20 \mathrm{~mm}$ aperture and integrated gradient of $2.435 \mathrm{Tm} / \mathrm{m}$, measured by a stretched wire system [14], with 
an accuracy of $\pm 0.002 \mathrm{Tm} / \mathrm{m}$. The magnetic measurement was carried out by moving the magnet along the longitudinal axis to evaluate the coil uniformity. The external coil ABS presents in the central part a strong variation in the width profile, probably due to the low rigidity of the small frame. The coil CMP is nested into the coil ABS, thus its width is more uniform, nevertheless the radius variation respect to the axis rotation reduces the uniformity of the measurement. The variations of the gradient measurement along the $200 \mathrm{~mm}$ coil length are about $5 \%$ for coil ABS and of $3 \%$ for coil CMP. This problem seems to be due to insufficient geometrical stability of the flat coil winding supports, which are made of G10 machined to a thickness of about $1 \mathrm{~mm}$. For this reason, a new shaft with an optimized compensation scheme for dipole and quadrupole is under development. On the other hand, an in-situ calibration method for the two coils, allowing an accuracy of $\pm 0.1 \%$ in the measurement of the magnetic gradient despite the geometrical imperfections, was developed.

In Figure 4, the harmonic field content measured on the prototype permanent quadrupole is shown. The modules of the harmonic coefficients (2) are expressed as a percentage of the main field component (in this case the quadrupole). The accuracy of the system up to the $10^{\text {th }}$ harmonic is better than $\pm 1 \%$, as required. Some systematic errors have to be investigated, which could affect the sextupolar field component measurement.

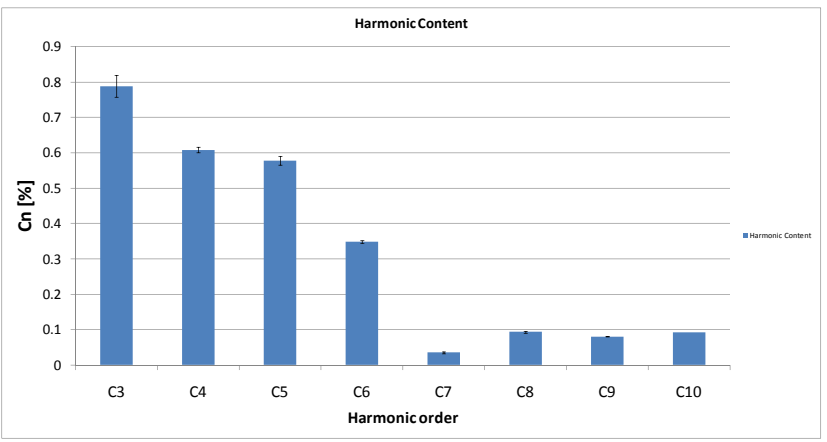

Figure 4: harmonic analysis of a permanent quadrupole

\section{CONCLUSIONS}

In this paper, a new system for fast pulsed and permanent quadrupole measurements is presented. The preliminary results show that the positioning system performs according to the specifications, although the quality of the coil construction should be improved, because the measurements present a strong variation along the whole coil length. After the calibration in a particular longitudinal position of the two coils an accuracy better than $\pm 0.1 \%$ for the magnetic strength measurement and $1 \%$ for the higher order harmonics can be reached. The measurement system still presents systematic errors to be removed by an improved measurement procedure and improved hardware (shaft, system components, ect.).

\section{ACKNOWLEDGMENTS}

Authors thank Ricardo Beltron Mercadillo, Felice Cennamo, Dominique Cote, Lucette Gaborit and Alessandra Lombardi for their useful suggestions and contribution.

\section{REFERENCES}

[1] R. Garoby, G. Bellodi, F. Gerigk, K. Hanke, A.M. Lombardi, M. Pasini, C. Rossi, E.Z. Sargsyan, M. Vretenar, "Linac4, a new injector for the CERN PS Booster", Proceedings of European Particle Accelerator Conference 2006, Edinburgh

[2] M. Kobayashi, S. Yamashita, "Measurement of harmonic field contents of pulsed magnets using a stepping search coil",Nuclear Instrument and Methods vol.103, pp 493-500,1972.

[3] P.Wanderer, J. Escallier, G. Ganetis, A. Jain, W. Louie, A. Marone, R. Thomas,"Development of a precise magnetic field measurement system for fast-changing magnetic fields", IEEE transactions on applied superconductivity, vol. 14, n. 2, June 2004.

[4] A. Jain, J. Escallier, G. Ganetis, W. Louie, A. Marone, R. Thomas, P. Wanderer,"Magnetic field measurement for fast-changing magnetic fields", IEEE Transactions on applied superconductivity, vol. 15, n. 2, June 2005.

[5] T. Zickler et al, "MAGNETS", internal note, October 2009.

[6] A. Jain, "Harmonic coils", CAS on measurement and alignment of accelerator and detector magnets, CERN 98-05, August 1998.

[7] P. Arpaia, A. Masi, G. Spiezia,'Digital integrator for fast accurate measurement of magnetic flux by rotating coils", IEEE Transactions on instrumentation and measurement, vol. 56, n. 2, April 2007.

[8] D. Giloteaux, D. Cote," Etude et conception d'un nouveau banc de mesures magnétiques à but général”, internal note, June 2007.

[9] P.Arpaia, L. Bottura, M. Buzio, D. Della Ratta, L. Deniau, V. Inglese, G. Spiezia, S. Tiso, L. Walckiers, "A software framework for magnetic measurement at CERN", Instrumentation and measuring technology conference- IMTC 2007, Warsaw, Poland.

[10] L. Bottura, " Standard analysis procedures for field quality measurement of the LHC magnets part I: Harmonics”, internal note, February 2001.

[11] L. Walckiers, "The harmonic-coil method",CERN Accelerator School on Magnets, June 1992, Montreux,Switzerland.

[12] R. M. Main, J.T. Tanabe, K. Halbach, "Measurement and Correction of the PEP Interaction Region Quadrupole Magnets", IEEE Transactions on nuclear science, vol. NS-26, No. 3, June 1979.

[13] O. Dunkel "Coil Manufacture, Assembly and Magnetic Calibration Facility for Warm and Cold Magnetic Measurements of LHC Superconducting Magnets", 14th International Magnetic Measurement Workshop- IMMW14, September 2005, Geneva, Switzerland.

[14] ] N. Smirnov, L. Bottura, M. Calvi, G. Deferne, J. DiMarco, N. Sammut, and S. Sanfilippo, "Focusing Strength Measurements of the Main Quadrupoles for the LHC", IEEE Transactions on Applied Superconductivity, vol. 16, no. 2, June 2006. 